that I have said nothing as to drugs. They are, indeed, of little consequence and the patient has doubtless already swallowed them in bulk. The only ones I ever give are some iron and arsenic, some aperient if required at first, and a tablespoonful of maltine two or three times daily.

'l'he psychological aspect of the "rest cure" is as important as the physical and the one is necessarily interdependent on the other. You must bear in mind how all these chronic invalids have fallen into morbid grooves in the conduct of their lives. Almost always there is some injudicious relative and friend who, is, as it were, the slave of the patient, and who, generally from the highest motives of love and sympathy, unconsciously fosters all her evil habits. These motives, however, although right, are generally injudicious. To attempt to carry on the case under conditions in which the friends are in constant communication with the patient is to court failure. I am not prepared to say that a case can never be cured at home, but it is so seldum that the exception only proves the rule, and no wise man will run the risk. I have alreary hinted at some of the personal qualities which the medical man ought to possess if he is to conduct these cases successfully. Every case should be the subject of a careful psychological study. No two cases are the same, no two can be managed in the same way. Beyond any doubt I think there is a good deal of suggestion in this business. If the patient comes with the profound conviction that she is to be cured, whether she likes it or not, then the battle is half gained, but how that confidence and conviction are to be given to the patient it is not in my power to tell you. All I can say on this is that in nine cases out of 10 it can be done. But if you think that you can pull one way while you leave relatives, friends, and visitors to pull the other at their own sweet will by a word, a hint, or a look, then you will soon find out your mistake. Therefore $\mathrm{I}$ would insist most strongly that complete separation from ordinary surroundings, as in a suitable home or lodgings, should always be made an absolute sine quâ non. Even letters should be prohibited, at least at first. If after a week you find your patient doing well, then this rule may be safely relaxed. I generally keep my cases rigidly confined to bed for about six weeks; then I get them up for some hours and I set them to work with the nurse to go out daily, to walk an increasing amount, to go to theatres, churches, and concerts - in fact, to do whatever was impossible before and what is incompatible with the life of an invalid. Then, if the patient's means admit of it, I send her away with the nurse to travel abroad, to go on a sea voyage, or to the seaside; or to do whatever is most likely to suit the particular case, and when the patient has been got to walk five or six miles daily, to ride on horseback, to go out bicycling, and so on, then no one can say that she is an invalid; but I would not advise the patient in any bad case to go home to her former surroundings unless she has passed through some such tests as I have indicated. And when once she has done so the cure may be considered to be established. I have often heard objectors say that it is no use curing the patients in cases of this type-that the improvement will be only transient and that very soon they will be as bad as ever. Now, that is certainly not the case. Of course, it would be absurd and irrational to expect that no case ever relapses, but all I need say on this point is that my experience, which I can now claim to be very large, has convinced me that any real and permanent relapse is quite an exception and may be considered as a negligible quantity. Of course, every now and again it must happen, but its possibility should never be a ground for not trying to get the patient out of the slough in which she is lying. Should a patient show any symptom of recurrence then the proper thing to do is to try to get her away from home for a week or two so as to nip the threatened danger in the bud. The one great objection that can be raised against this system of treatment is the very consiclerable expense that is necessarily associated with it and which I have never seen my way to get over. I once tried to treat cases in a private room at King's College Hospital, but they were found to be so costly to the institution, as well as troublesome, that the hospital authorities would not allow me to take in any more. I am afraid that any attempt to treat them cheaply will end in failure. All I can say on this is that there is nothing so expensive to a family as constant ill-health and that, therefore, the expenditure will generally be found to be a real economy in the long run.

\section{THE OCCURRENCE OF GREEN OR BIUE URINE AND ITS MOST FREQUENT CAUSE}

By F. PARKES WEBER, M.D. Cantab., F.R.C.P. Lond., Physichan to the grerman fospilia, Dalston.

So much has been written, especially in France. on the effects of the ingestion of methylene blue on the urine, that one would think that there was little more to be said on the subject. It happens occasionally, however, that apfarently healthy subjects, mostly children, pass green or blue urine, though they are unaware of having swallowed methylene blue or any other similar anilin dye in any form. Medical men not familiar with the characteristic properties of methylene blue and the reactions of urine coloured with methylene blue are therefore very apt to seek some other explanation-for instance, such an unusual one as that indigo blue, derived by fermentation from indican, is present in the urine before it leaves the body. As a matter of fact, apart from the greenish urine resulting from the presence of biliverdin and apart from the somewhat greenish urine of carboluria, practically all cases in which green or blue urine is voided are due to the ingestion of methylene blue or a similar anilin dye, though some poisoncus plants may make the urine green and Zülzer ${ }^{1}$ recorded the case of a boy who became severely ill after eating the leaves of Cytisus alpinus and passed a large quantity of urinehaving a grass-green colour. I am even sceptical of the power of indigo, given medicinally or otherwise, to colour the urine green. At all events, whereas a capsule containing one decigramme of methylene blue will make the urine green or blue during the next three or four days, several doses of the same amount of indigo have no effect of this kind, the colour of the urine remaining unaltered or simply becoming yellower or more decidedly orange. I think that when apparently healthy rersons in England pass green or blue urine without obvious cause the real explanation is generally that they have indulged in sweets coloured with methylene blue, rarticularly certain rurple sweets. These latter contain a mixture of dyes and the urine passed after eating them is greenish. "Thus, the supposed "unaccountable" secretion of green urine generally occurs in children and young women who like sweets. As a considerable quantity of sugar has to be swallowed for the urine to become deeply coloured (other kinds of sweets are likely to be eaten at the same time as those containing methylene blue) it is not surprising that some of those who secrete green urine should suffer from digestive troubles and a general metabolic disturbance, the latter showing itself by a sediment of uric acid or calcium oxalate ${ }^{2}$ crystals in the urine. These disagreeable effects are certainly not so readily produced by swallowing pure methylene blue (medicinally) in considerably greater amount than that contained in the sweets.

Last April a practitioner gave me a srecimen of green urine from a woman, aged 28 years, who had been passing similar urine for four days. The ordinary tests for methylene blue gave positive results, and though the patient was not aware of having taken methylene blue in any form she had, as she acknowledged, been eating sweets. The urine was of specific gravity 1023, acid, free from sugar and albumin, but loaded with small uric acid crystals. Perhaps some hepatic disturbance due to indulgence in sweets accounted for the uric acid sediment. Later, in June, through the kindness of Dr. A. E. Garrod, I obtained a specimen of bluish-green urine from a girl, aged nine years, together with a sample of sweets similar to those which she had been eating. The urine was of specific gravity 1022, acid, and free from albumin and sugar, but after standing it deposited a sediment of oxalate of calcium crystals. The ordinary tests

1 Harn Analyse, p. 54, quoted by Dr. H. Morley Fletcher, Transactions of the Clinical Society of London, 1899, vol. xxxii., p. 49.

2 There is no cloubt that oxaluria may sometimes be regarded as a forerunner of alimentary glycosuria-that is, as being due to the same kind of metabolic disturbance which when more rue to the same itself by the preserce of sugar in the urine. See Dr. Paul Mayer's. itself by the preserce of sugar in the urine. See Dr. Paul Mayer's excellent paper, "Ueher unvollkommene Zuckeroxydation im Organismus," Deutsche Medicinische Wochenschrift, 1901, Nos. 16 and without sugar may in some individuals produce or increase oxaluria. 
showed that the colour was due to methylene blue. The sweets in question were violet or purple coloured, becoming opaque and more reddish on keeping. 'Ihree or four pieces swallowed experimentally sufficed to impart a green colour to the urine passed two or three hours afterwards; this green colour was shown by the ordinary tests to be due to methylene blue. In July a woman who attended as an out-patient at the North London Hospital for Consumption told me that her daughter, six years old, had recently had a peculiar affection. During about eight weeks the child from time to time passed greenish urine. The green colour used to be observed for two or three days consecutively every week, and only in the urine passed in the morning. I ascertained that the child had been occasionally eating purple-coloured sweets, though she preferred others when she had the choice. In this case I succeeded in getting some of the purple sweets from the maker. They were similar to those mentioned in the previous case, and after experimentally eating some of them greenish acid urine was excreted, giving the ordinary proofs of the presence of methylene blue.

I have taken considerable trouble to test the accuracy of various accounts of the characters by which a green or blue colouration of the urine may be recognised as due to methylene blue. Some mistakes in this connexion have been published, but I believe the following summary to be correct.

1. When a capsule containing one decigramme of methylene blue has been swallowed by a fairly healthy adult the urine passed an hour or so afterwards is already found to have a greenish tint. 'l'he urine voided later is deeper green, and still later it is blue. The colour is observed in the urine of the next three or four days, but becomes less intense and finally intermittent, the earliest morning urine being the best coloured. In some individuals a green tint may even be observed in the morning urine after five days, though the midday urine of the two preceding days has only the ordinary yellow appearance. This accounts for the history sometimes given that the green colour has been observed chiefly, or exclusively, in the morning urine-that is, in the urine secreted during the night's sleep. Thus when relatively small doses of methylene blue in the form of sweets are taken at intervals during several weeks, as in the third case which I have alluded to, it is not surprising to hear that during eight weeks the green was noticed only in the morning urine.

2. The colour of the urine varies from faint greenishyellow to deep blue and depends, of course, on the amount of methylene blue and of the ordinary yellowish pioment present in the specimen. The urine quickly stains ordinary white writing- or blotting-paper blue.

3. The colour of the urine may sometimes be increased by boiling. This is because some of the methylene blue is excreted as a colourless "chromogen." The colour is brought back by heating the urine with acetic acid, as Achard and Castaigne ${ }^{3}$ say, or, in acid urine at all events, as $I$ have noted, merely by boiling it. The amount of methylene blue secreted as a colourless chromogen is very much greater in some animals (the dog, the rabbit, and the guinea-pig) than in man, and is increased by the presence of certain pathological conditions in man.

4. The colour of the urine is slightly lessened by filtering it. This is because part of the methylene blue is absorbed in staining the filter paper, and sometimes also because the urine contains suspended uric acid crystals stained with the blue which cannot pass through the filter paper.

5. The urine is decolourised by adding a solution of caustic potash without heating. A few drops of nitric acid will not effect this, but decolourisation can generally be effected by boiling with strong nitric or hydrochloric acid.

green or blue can be bronght back by neutralisation.

6. Ether, when shaken up with the urine, does not become coloured, but chloroform takes up much of the dye and rapidly becomes blue. This it will not do with an ordinary aqueous solution of methylene blue. Generally, when shaken up with the latter, chloroform gradually becomes mauve, and afterwards purple or red, causing some diffuse absorption in the green of the spectrum. ${ }^{*}$ It is probable, however, that the anilin dyes which after passing through the body appear in the mine as methylene blue are not all quite identical with each other-in other words, that

${ }^{3}$ Sur la Décoloration du Bleu de Méthylène par les Élements Vivants, Comptes rendus de la Société de Biologie, Paris, 1897, p. 1091. $4 \mathrm{Br}$ spectroscopic examination of the rendish chloroform a methyleneolue absorption band can sometimes also be maile out. there are different varieties of anilin dyes $^{5}$ resembling methylene blue which when absorbed from the alimentary canal are excreted in the urine as methylene blue. The blue colouration of chloroform by the methylene blue in the urine does not absolutely prove that the anilin dye has passed through the body, for outside the body ordinary methylene blue if dissolved in any urine, whether freshly passed or otherwise, becomes rapidly capable of giving the characteristic blue colour to chloroform.

7. On examining the green or blue urine with a good pocket spectroscope an absorption band can sometimes, but not always, be made out, identical with that yielded by an aqueous solution of methylene blue. The absorption band can, however, be best examined in a pale blue chloroform solution derived from the urine. It is a clearly defined band in the red of the spectrum separated by a considerable space from the sodium band (Frauenhofer D), whereas the characteristic band from a chloroform solution of indigo (and of artificial indigo) is further from the red end of the spectrum and quite close to the sodium band. In more deeply coloured solutions of methylene blue there is a second band in about the position of the indigo band, and in still stronger solutions the two bands united spread out from the red into the yellow and green of the spectrum. If a greenish colour in urine is due to indigo blue the chloroform should take on a bluish tint somewhat different from that due to the presence of methylene blue, and if the chloroform becomes sufficiently coloured the characteristic absorption band of indigo should be detected whilst that of methylene blue should be absent; moreover, when indigo is present the urine is likely to be decomposing and alkaline and by microscopical examination of the sediment the blue granules of the pigment will probably be seen. ${ }^{6}$

8. Methylene blue is reduced and decolourised by the vital action of living organisms. Consequently when urine containing methylene blue begins to ferment the lower portion of the liquid, owing to the action of the reducing microbes, loses its colour and only the upper surface retains it. 'There the anilin dye forms a thin blue or green film which cannot be decolourised because it is directly exposed to the oxygen of the air. On gently shaking up the liquid and thus mixing it with oxygen the colour may be seen rapidly spreading downwards from the surface until the original appearance of the whole is regained. If the decomposing urine is in a tightly-corked bottle, but does not quite fill it, this beautiful experiment may be repeated several times; but at last all the oxygen in the bottle is used up by the reducing microbes and the urine by reduction entirely loses its colour. Then no amount of shaking will bring back the colour until the cork be withdrawn so as to let out the carbonic acid and admit oxygen. On taking such a bottle of decolourised fermenting urine out of a cupboard and withdrawing the cork and pouring out the liquid into a glass the colour rapidiy returns and the erroneous conclusion may be arrived at that it is exposure to light instead of oxygenation which causes the colour to return. Such a mistake has been made, but Achard and Castaigne ${ }^{7}$ drew attention to this decolourising effect of living organisms on methylene blue as far back as in 1897. The same action of living tissues explains why the ingestion of a considerable dose of methylene blue scarcely alters' the colour of the living human blood and tissues. The methylene blue, until it is excreted by the kidneys, is present in the body as a

5 At all events $I$ find that chloroform when added to an aqueous solution of the purple sweets (from the thirc case mentioned previously) remains uncoloured, though it takes on a mauve or purple colour when shaken up with an aqueous solution of orlinary methylene blue. Yet the green urine obtained by eating these sweets gives the characteristic blue colour to chloroform, just as does any green urine from the merlicinal use of methylene blue. Horeover, on dissolving some of the sweets in a glass of urine and adding chloroform the latter at once becomes blue.

6 I am indebted to Mr. J. Good of Stockport for an example of such urine. It was from an old lady, aged 68 years, who sait that it was bright green when voiler and that she had never noticed her urine of that colour before. When I examined the specimen, about ten days afterwards, it still har a greenish colour, but was turbid, alkaline, and decomposing, and there was a bluish sediment in which blue granules could be seen under the microscope, as well as some triple phosphate crystals, \&e. Chloroform shaken up with the sediment took on a faint blue colouration. This was intensified by heating and evaporating off part of the chloroform, and then by spectroseopic examination the characteristic absorption band of indigo blue was observer. This ease is, of course, not the one descriled br Mr. Good in THE LANCET of June that the colour of the urine was due to methylene blue. that the colour of the urine was rine to methrlene bluc. 
colourless chromogen - that is to say, in its reduced form. Dr. L. Hill has made use of the dye to show how oxygen is used up by cerebral work, the methylene blue being reduced and decolourised by the activity of the brain substance.

Naturally the decolourisation of green urine beginning to ferment may be delayed or prevented by boiling it or adding glacial acetic acid, or chloroform, or any antiseptic preservative substances. The following experiment well illustrates the action of living organisms on methylene blue. If to a weak solution of sugar coloured by methylene blue a little yeast is added the blue colour gradually disappears from the lower layers of the liquid as fermentation progresses. When the sugar is used up fermentation ceases and the blue colour returns, because the yeast cells though still alive have nothing to do. When, however, fresh sugar is added the yeast cells regain their activity and reduce the methylene blue to the state of a colourless chromogen.

The question of green urine from eating coloured sweets is not without its practical importance, for a suspicion of the true cause may save a good deal of time and possibly some anxiety. A medical man when he prescribes methylene blue should not forget, for obvious reasons, to warn the patient of the effect of the drug on the urine, and vendors of sweets, as they can hardly converse with their customers on urinary topics, ought certainly never to sell any sweets containing the colouring matter in question.

Though fuchsin, when given as medicine-as it frequently was some years back for renal affections-quickly reddens the urine I have never heard of a case in which the urine has been thus coloured from eating red sweets. Probably, therefore, fuchsin is not employed as one of the substitutes for cochineal. An eosin-like anilin dye is, however, sometimes employed for sweets. In 1898 the urine of a child just admitted to the German Hospital was found to be markedly fluorescent, somewhat like a weak solution of eosin, appearing pink by transmitted light and golden green when viewed against a black background. About three days after admission the urine was slightly acid and of specific gravity 1020 and still looked greenish-yellow against a black background, though the pink colour by transmitted light had disappeared. Dr. A. E. Garrod kindly told me of a similar case from eating anilin-dyed sweets and I obtained samples of coloured sweets which the child had partaken of just before admission to the hospital. Amongst them were pink discs like "musk-drops" or "rose-drops," and these when dissolved in water yielded a solution fluorescent like the child's urine. The child had doubtless eaten very many of the sweets, for a few only seem not to colour the urine. This is probably why similar urine is seldom observed.

Harley-street, W.

\section{TWO CASES OF BLACKWATER FEVER.}

\section{BY FREDERICK SMITH, L.R.C.P. \& S. IREL.,} CAPTAIN, R.A.M.C.

\section{With Pathological Notes by}

\section{LOGAN TAYLOR, M.B., CH.B. GLASG.,}

OF THE LIVERPOOL MALARIAL COMMISSION; LATE ASSISTANT TO PROFESSOR OF PATHOLOGY, GLASGOW UNIVERSITY.

THE paucity of complete post-mortem records of cases of the little-understood disease, blackwater fever, clinically observed from beginning to end, justifies, it is thought, the publication of this paper.

CASE 1. - The patient was a European who had been for over a year in Sierra Leone doing duty as a non-commissioned officer. He was an exceptionally robust and energetic man. He had not been on the sick-list during his tour, but said that he had "had a couple of hours' fever" occasionally. However, he was seen on the morning of the first day of his illness looking well, smoking, and getting about his work. At 3 P.M. he felt ill, shivered a little, vomited, had a pain in his back, and by tea-time he was passing hæmoglobinuric urine of the usual character. He stated that he had taken five grains of quinine three or four times a week. Next morning he passed about a pint of similar urine. The backache was now agonising and green bilious vomit was frequent. No more urine of any kind was passed for three days. Then he passed in two instalments five ounces containing albumin and none for another three days. The day before his death 12 ounces were removed by catheter in the morning and two ounces in the evening. It contained very little albumin. No hæmoglobin was apparent in the urine after the second day of illness. Jaundice was noticeable within a few hours of the onset of the disease and was intense by the third day. Repeated examinations failed to demonstrate malarial organisms in the blood. Treatment was with boric acid and arsenic-also, of course, symptomatically. During the last four days of illness I (F. S.) made intravenous injections of normal salt solution ranging from half a pint to one and a half pints twice a day by means of an irrigator and an aspirating needle, with a slight temporary good effect on the skin and perhaps the pulse. life was sustained mainly by nutrient enemata, almost everything given by the mouth being ejected. Vomiting, in spite of all treatment, remained persistent, the matter brought up being progressively darker until it attained a deep mahogany colour. Meantime, the jaundice became less marked and had almost gone before death. The mind was confused about the fourth day, but more or less mental clearness remained to the last. and the patient conversed intelligibly the night preceding his death. Hiccough was troublesome from time to time and there were twitchings of the fingers. 'The mouth during the last few days was much inflamed and there were hæmorrhages from the buccal mucous membrane. The saliva was blood-tinged and mingled with material of the same mahogany colour as the vomit. Sleeplessness was troublesome only for the first three days. The stools were scanty and loose, naturally bile-stained in colour, but containing no blood. The recorded temperature was never above $101^{\circ} \mathrm{F}$. but the temperature commonly dropped with the appearance of the hæmoglobinous urine and it was in this case not taken before owing to the sudden onset. Death took place on the tenth day.

Dr. Taylor made daily blood counts with the following: results. On July 24th, the first day of illness, no count was made. On the following dates the number of the red and white blood corpuscles per cubic millimetre was as follows: $25 \mathrm{th}$, red $1,900,000$ (24 hours after onset), white 10,000 ; $26 \mathrm{th}$, red $1,700,000$, white 10,$000 ; 27 \mathrm{th}$, red $1,000,000$ 28 th, red 900,000 ; 29th, red $1,100,000$, white 18,000 (increase chiefly polynuclear) ; 30th, red 1,000,000; and 31st, red $1,200,000$, white 10,000 . On August 1st no count was made. On the 2 nd death occurred.

Necropsy. - At the post-mortem examination the body was found to be that of a fairly muscular man. The skin and conjunctivæ were of a pale lemon-yellow tint. The lips and teeth were covered with sordes. On examination of the thorax the heart was found to be of normal size and weight. There was marked fatty infiltration of the wall of the right ventricle. The heart muscle was pale in colour and was very soft and friable, and beneath the endocardium of both ventricles there was a yellowish mottling which was most distinctly seen on the papillary muscles and was due to fatty. degeneration of the muscle. The cavities of the heart were of normal size and with the exception of slight dilatation of the mitral and tricuspid orifices the valvular apparatus was healthy. The aorta was also quite healthy. The lungs were free from adhesions and showed only slight hypostatic congestion and cedema at their bases. The peritoneum was normal in appearance and there was no fluid in the abdominal cavity. The spleen was firm and was considerably enlarged, weighing nine ounces, and the capsnle was adherent to the surrounding parts. It was very much congested, but the pulp was not at all diffluent and it was more of a walnut colour than is usually found in a simple congested spleen. The Malpighian bodies were slightly swollen and the trabeculæ were thickened. Microscopical examination of films made from the pulp showed that this walnut colour was due to a large amount of blood pigment. Great numbers of degenerated red corpuscles were also seen. The liver was enlarged, weighing 4 pounds 13 ounces, and was very soft. It was much congested and on section it presented a colour similar to that of the spleen pulp; there was also a yellowish mottling throughout which was due to fatty degeneration. The gall-bladder was distended with coagulated bile. The weight of the kidneys without their intimate capsules was-right nine ounces, left eight and a half ounces. Both presented a similar appearance, so that the one description will do for both. The cortex was enlarged and swollen, measuring fully three-quarters of an inch. Botb cortex and inter-pyramidal cortex were of the same walnut 